\title{
Estimating apparent motion on satellite acquisitions with a physical dynamic model
}

\author{
E. Huot ${ }^{1,2,3}$, I. Herlin ${ }^{1,2}$, N. Mercier ${ }^{1,2}$, E. Plotnikov ${ }^{4}$ \\ ${ }^{1}$ INRIA, Institut National de Recherche en Informatique et Automatique, France \\ ${ }^{2}$ CEREA, Joint Lab. École nationale des Ponts ParisTech-EDF R\&D, Université Paris-Est \\ ${ }^{3}$ Université Versailles St-Quentin, France \\ ${ }^{4}$ Marine Hydrophysical Institute, National Academy of Sciences, Sevastopol, Ukraine \\ Etienne.Huot@inria.fr, Isabelle.Herlin@inria.fr
}

\begin{abstract}
The paper presents a motion estimation method based on data assimilation in a dynamic model, named Image Model, expressing the physical evolution of a quantity observed on the images. The application concerns the retrieval of apparent surface velocity from a sequence of satellite data, acquired over the ocean.

The Image Model includes a shallow-water approximation for the dynamics of the velocity field (the evolution of the two components of motion are linked by the water layer thickness) and a transport equation for the image field. For retrieving the surface velocity, a sequence of Sea Surface Temperature (SST) acquisitions is assimilated in the Image Model with a 4D-Var method. This is based on the minimization of a cost function including the discrepancy between model outputs and SST data and a regularization term. Several types of regularization norms have been studied. Results are discussed to analyze the impact of the different components of the assimilation system.
\end{abstract}

\section{Introduction and state of the art}

Ocean surface velocity is approximated using image processing techniques on satellite image sequences. Methods for estimating a dense motion field usually rely on a conservation equation [5], which is however not sufficient to compute the two components of the velocity vector. This is named the aperture problem. A Tikhonov regularization is then applied, relying on regularity heuristics. In $[4,6]$, authors use a regularity constraint based on the irrotational and solenoidal description of the motion field. To apply the approach on fluid motion, authors $[2,4]$ also propose to replace the usual luminance conservation hypothesis by a mass conservation equation. However, these processing techniques have two main drawbacks. First, the algorithms rely on the computation of spatial and temporal derivatives, which is impossible if some data are occluded by clouds or contaminated by noise. Second, the equations have no physical origin. The image processing community got a recent interest in data assimilation tools $[1,3,8]$, which partly overcome the two previous concerns. We proposed such method in [7]: the available satellite images constitute observations and are assimilated into an Image Model (IM), in order to derive motion pseudo-observations, that are in turn assimilated in an operational oceanic model. This $I M$, named in the following Simple Image Model (SIM) and its limitations are summarized in section 2, in order to introduce the so-called Extended Image Model (EIM), described in section 3. The EIM expresses: ( $i$ ) the transport of temperature by surface velocity with a simplified version of the 3D advection-diffusion equation that applies to temperature transport in oceans; (ii) the dynamics of the surface velocity, whose components are linked by the water layer thickness, using the shallow-water approximation. Variational data assimilation is then applied to assimilate SST observations into EIM and estimate surface motion (section 4). Section 5 illustrates the results and discusses the impact of the different options of the data assimilation software.

\section{Simple Image Model}

We consider the advection-diffusion equation governing the transport of temperature $T$ by a 3D motion field $\mathbf{w}$ :

$$
\frac{\partial T}{\partial t}=-\nabla T \cdot \mathbf{w}-T \cdot \operatorname{div} \mathbf{w}+K_{T} \Delta T+S r c-S n k .
$$

$K_{T}$ denotes the temperature diffusivity, $S r c$ and $S n k$ are the source and sink terms corresponding to the local heat flow. Equation (1) is simplified: $(i)$ it is assumed to be valid for the 2D motion field $\mathbf{v}$; (ii) water is assumed incompressible $(d i v \mathbf{v}=0)$; (iii) $S r c$ and $S n k$, are neglected. Hence, the evolution of temperature is modelled in $2 \mathrm{D}$ by:

$\frac{\partial T}{\partial t}=-\nabla T \cdot \mathbf{v}+K_{T} \Delta T$.

We assume that the surface velocity field $\mathbf{v}$ evolves much slower than the temperature field. This heuristic is acceptable for a large range of marine processes. If a vortex, whose 
spatial scale is more than $10-50 \mathrm{~km}$ is transported with a velocity less than 0.1 to $0.5 \mathrm{~m} / \mathrm{s}$ then the temporal scale of that phenomenon will be more than one day. It means that the surface velocity field can be considered as stationary during one day, i.e.: $\frac{\partial u}{\partial t}=\frac{\partial v}{\partial t}=0$, if the motion vector is written $\mathbf{v}=(u, v)^{T}$.

SIM is composed by the previous equations. Satellite acquisitions are assimilated into SIM in order to retrieve surface velocity. The main limitation concerns the heuristic on velocity, which is not always valid. Consequently SIM is only applicable for a short temporal window, typically 12 hours, without any non linear phenomena. The remaining of the paper describes a more accurate physical model and its assimilation system.

\section{Extended Image Model}

The issue of ocean modelling has long been addressed by oceanographers and applied mathematicians. In this paper, the Saint-Venant formulation is considered. It approximates the Navier-Stokes equations in 2D, leading to shallow-water models used for atmospheric simulation, hydrology and operational oceanography. The equations link the $2 \mathrm{D}$ velocity $(u, v)$ of a merged layer to its thickness $h$ or, more precisely, to the thickness anomaly, i.e. $\eta=h-h_{m}$, with $h_{m}$ denoting the average value.

Including the transport equation of temperature, the $E x$ tended Image Model (EIM) is:

$$
\left\{\begin{array}{l}
\frac{\partial T}{\partial t}=-u \frac{\partial T}{\partial x}-v \frac{\partial T}{\partial y}+K_{T} \Delta T \\
\frac{\partial u}{\partial t}=-u \frac{\partial u}{\partial x}-v \frac{\partial u}{\partial y}+f v-g^{\prime} \frac{\partial \eta}{\partial x}+K_{\mathbf{v}} \Delta u \\
\frac{\partial v}{\partial t}=-u \frac{\partial v}{\partial x}-v \frac{\partial v}{\partial y}-f u-g^{\prime} \frac{\partial \eta}{\partial y}+K_{\mathbf{v}} \Delta v \\
\frac{\partial \eta}{\partial t}=-\frac{\partial(u \eta)}{\partial x}+\frac{\partial(v \eta)}{\partial y}-h_{m}\left(\frac{\partial u}{\partial x}+\frac{\partial v}{\partial y}\right)
\end{array}\right.
$$

$f$ denotes the Coriolis parameter; $K_{\mathrm{v}}$ the viscosity of the water layer; $g^{\prime}=g\left(\rho_{0}-\rho_{1}\right) / \rho_{0}$ the reduced gravity. $\rho_{0}$ corresponds to the reference density and $\rho_{1}$ to the average density of the water layer. EIM includes a physical description of the motion dynamics which permits to overpass the previous limitations of SIM. It allows to process large assimilation windows (more than one day), even those displaying non linear effects.

\section{Data assimilation}

The aim is to assimilate a sequence of SST acquisitions into the Image Model and estimate the surface velocity over a temporal window $\tau=\left[t_{0}, t_{N}\right]$. A set of images $\left\{Y\left(t_{1}\right), \ldots, Y\left(t_{N}\right)\right\}$, acquired at dates $t_{1}$ to $t_{N}$ and over the spatial domain $\Omega$, are used as observations.

\subsection{Variational approach}

The system (2) is summarized as: $\frac{\partial X}{\partial t}=F(X)$, with $X(t)=(T(t), u(t), v(t), h(t))^{T}$ the model state vector and $F$ the evolution model corresponding to the right hand side.

The links between $X(t)$ and the observation $Y(t)$ are expressed through the observation operator $H: Y(t)=$ $H(X(t))+\varepsilon_{o}, \varepsilon_{o}$ stands for the observation noise. This equation is only valid at acquisition dates $t_{1} \ldots t_{N} . H$ is a simple projection operator, because surface temperature is the first component of the state vector. $\varepsilon_{o}$ is assumed to be Gaussian and characterized by its covariance matrix $\mathbf{R}$.

The background value of $X\left(t_{0}\right)$ is supposed to be $X_{0}$. This provides the equation $X\left(t_{0}\right)=X_{0}+\varepsilon_{b}$. $\varepsilon_{b}$ is the error on the background value. It is assumed to be Gaussian and characterized by its covariance matrix $\mathbf{B}$.

The cost function $J$, which has to be minimized with respect to the control variable $X\left(t_{0}\right)$ :

$$
\begin{aligned}
& J\left(X\left(t_{0}\right)\right)= \\
& \quad \int_{\Omega, \tau}(H(X)-Y)^{\mathrm{T}} \mathbf{R}^{-1}(H(X)-Y) d x d y d t \\
& +\int_{\Omega}\left(X\left(t_{0}\right)-X_{0}\right)^{\mathrm{T}} \mathbf{B}^{-1}\left(X\left(t_{0}\right)-X_{0}\right) d x d y .
\end{aligned}
$$

The first term of (3) expresses the discrepancy between the model outputs and the observations and the second term the confidence on the background. One can refer to [1] for a discussion about the choice of the matrix $\mathbf{R}$ and its impact on the result. The variational data assimilation approach consists in minimizing $J$ and estimating $\hat{X}_{0}$, that verifies $\hat{X}_{0}=\operatorname{argmin} J\left(X\left(t_{0}\right)\right)$ or equivalently $\nabla J\left(\hat{X}_{0}\right)=0$ $\left(\hat{X}_{0}=\left(\hat{T}_{0}, \hat{u_{0}}, \hat{v_{0}}, \hat{h_{0}}\right)\right)$.

The result $\hat{\mathbf{v}_{0}}=\left(\hat{u_{0}}, \hat{v_{0}}\right)^{T}$ is then considered as the estimation of motion at $t_{0}$ and its integration over $\tau$ provides the space-time motion field.

\subsection{Regularization}

As stated in the introduction, a regularization term is added to $J$ in order to obtain a convex cost function: the minimization process converges to a global minimum. Several heuristics have been compared:

1. $R_{1}=\int_{\Omega} \alpha\left(|\nabla u|^{2}+|\nabla v|^{2}\right) d x d y+\int_{\Omega} \beta d i v \mathbf{v} d x d y$

The integral is spatial, because the constraint is only applied at $t_{0}$.

The first term of $R_{1}$ penalizes the variation of the norm of motion and the second one relies on the incompressibility assumption and penalizes the value of the divergence. $J_{1}=$ $J+R_{1}$ denotes the cost function built from equations (3) and (4).

2. $\quad R_{2}=\int_{\Omega}\left[\alpha|\nabla \operatorname{div} \mathbf{v}|^{2}+\beta|\nabla \operatorname{curl} \mathbf{v}|^{2}\right] d x d y$ 
The fluid motion being described by its irrotational, $\operatorname{div} \mathbf{v}$, and solenoidal, curl $\mathbf{v}$, components, $R_{2}$ is used to penalize their variations. $J_{2}=J+R_{2}$ denotes the cost function built from equations (3) and (5).

\section{Impact of the data assimilation options}

The first concern is to demonstrate the potential of EIM. For that purpose SIM and EIM have been both applied on a sequence of satellite data. Results are displayed on figure 1. The first line shows the constant motion field computed by SIM: it is represented by black vectors superposed to image data. On the second line, the three consecutive motion fields retrieved by EIM allow to visualize the vortex evolution.
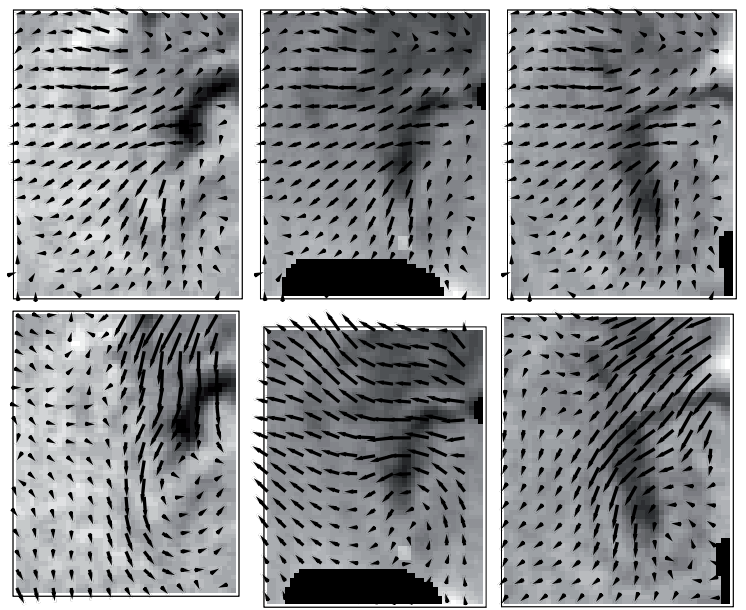

Figure 1. Motion estimation superposed to satellite images. Top: SIM results, bottom: EIM results.

For better understanding the options of the data assimilation algorithm, we discuss the results on twin experiments. The synthetic images, simulating SST data and further used as observations $Y$, are obtained by integrating EIM from known initial conditions: $\overline{X_{0}}=\left(\overline{T_{0}}, \overline{u_{0}}, \overline{v_{0}}, \overline{h_{0}}\right)^{T}$ displayed on figure 2. Five observation images are considered: the first one
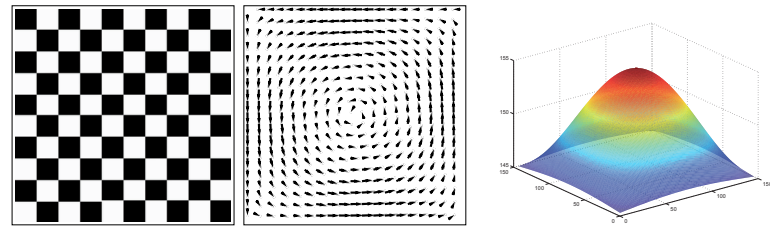

Figure 2. Left: temperature image. Center: velocity field. Right: 3D visualization of the thickness. at $t_{1}=t_{0}+d t$, and the others with a regular time interval of $200 d t$. dt being the time step of the simulation process. Three observations are illustrated on figure 3 . The experiment

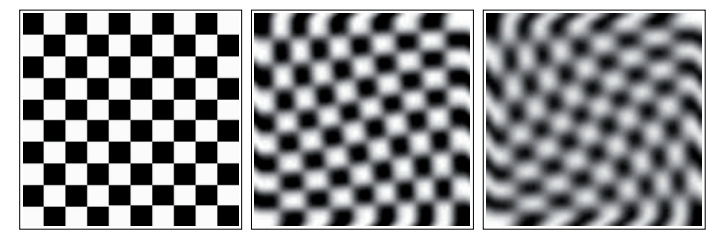

Figure 3. Simulated observations.

consists in assimilating the observations into EIM and compare the result $\hat{X}_{0}$ to the reference $\overline{X_{0}}$, The parameters are: the cost function ( $J_{1}$ or $J_{2}$ ), the regularization weights $\alpha$ and $\beta$, and the background value $X_{0}$. We set fixed values to $\alpha$ and $\beta$ and only discuss the impact of the initial conditions and the cost function.

\subsection{Impact of the background thickness field $h_{0}$}

We first set $X_{0}=\left(T_{t_{1}}, 0,0, \overline{h_{0}}\right)^{T}$ :

- The temperature field $T_{0}$ is initialized with the first observation $T_{t_{1}}$;

- We assume no knowledge on the motion field; $\mathbf{v}_{\mathbf{0}}=$ $\left(u_{0}, v_{0}\right)^{T}=(0,0)^{T}$;

- We consider having a perfect knowledge of the thickness anomaly; $h_{0}=\overline{h_{0}}$.

The motion results obtained with $J_{1}$ and $J_{2}$ at $T_{0}$ are displayed on figure 4 . In this case, the quantitative analysis

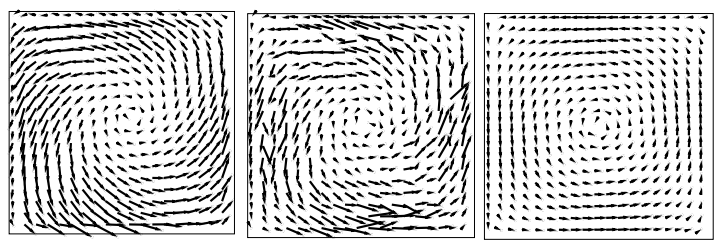

Figure 4. Left: with $J_{1}$. Center: with $J_{2}$. Right: truth.

demonstrate that both the norm and the direction of the motion vector are better estimated with $J_{2}$ (average norm error less than $1 \%$, average angular error approximately 5 degrees).

In a second experiment, we use no prior knowledge on the interface and the background is set to $h_{0}=h_{m}, h_{m}$ being the average value. In operational cases, this average value is known from ocean specialists. The results are similar to those obtained with a perfect knowledge of $h_{0}$ and the conclusions on errors are similar. This remark allows applying the software on real data, without having to perform a complex preprocessing to get an accurate thickness background value. 


\subsection{Impact of the background motion field $v_{0}$}

This analysis concerns the background value for the motion field $\mathbf{v}_{0}$. Different values have been obtained from image processing algorithms. In the following, the comparison concerns a null motion field, $\left(u_{0}, v_{0}\right)=(0,0)$, and one obtained by applying the Horn and Schunk method [5] on the first two frames (result is on figure 5). In both cases, the two other background values are $T_{0}=T_{t_{1}}$ and $h_{0}=h_{m}$, and data assimilation is performed with the cost function $J_{1}$.

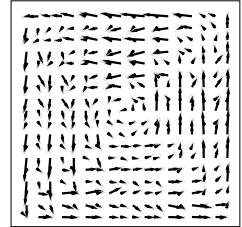

Figure 5. Result on the first two frames from the Horn and Schunk method.

The results are displayed on figure 6. That demonstrates

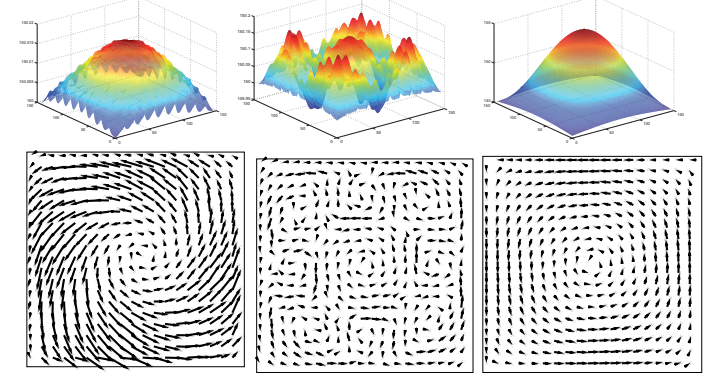

Figure 6. Left: result of estimation with a null motion background; center: with a background motion estimated with the Horn \& Schunk method; left the ground truth.

that a null initial condition allows a better estimation than a non perfect initialization, which can be incompatible with the whole image sequence, because computed only on the first two frames.

\section{Conclusion}

This paper presents a motion estimation method relying on image assimilation in an Image Model. The IM includes some physical knowledge on the dynamics and is based on the shallow-water equations. The two components of velocity are linked by the thickness of the water layer. The 4D-Var assimilation is performed and equivalent to minimizing a cost function, that expresses the discrepancy between image observations and model outputs and includes a regularity component.

The data assimilation system has a number of options: regularization term, parameters values, initial conditions. Their impact is analyzed with results obtained on synthetic data in order to quantify the differences. It has been shown that the best results are obtained with the shallow-water evolution equations, with null background value for the motion field, constant background value $h_{m}$ for the thickness field, and a regularization term that penalizes the variations of the divergence and the rotational.

\section{Acknowledgments}

This work has been performed in the context of the ADAMS associated-team (program launched by INRIA). It has been done in collaboration with the Marine Hydrophysical Institute of Sevastopol, Ukraine. We are very thankful to Pr. Korotaev for his strong knowledge on oceanic science.

\section{References}

[1] D. Béréziat and I. Herlin. Solving ill-posed problems using data assimilation - Application to optical flow estimation. In VISSAPP, pages 595-602, 2009.

[2] D. Béréziat, I. Herlin, and L. Younes. A generalized optical flow constraint and its physical interpretation. In CVPR, pages 2487-2492, 2000.

[3] T. Corpetti, P. Héas, E. Mémin, and N. Papadakis. Variational pressure image assimilation for atmospheric motion estimation. In IGARSS, volume 2, pages 505-508, Boston, MA, July 2008.

[4] T. Corpetti, E. Mémin, and P. Pérez. Dense estimation of fluid flows. IEEE Transactions on Pattern Analysis and Machine Intelligence, 24(3):365-380, March 2002.

[5] B. Horn and B. Schunk. Determining optical flow. Artificial Intelligence, Vol 17:185-203, 1981.

[6] T. Isambert, J.-P. Berroir, and I. Herlin. A multi-scale vector spline method for estimating the fluids motion on satellite images. In ECCV, pages 665-676, 2008.

[7] G. Korotaev, E. Huot, F.-X. L. Dimet, I. Herlin, S. Stanichny, D. Solovyev, and L. Wu. Retrieving ocean surface current by 4-D variational assimilation of sea surface temperature images. Remote Sensing of Environment, 112(4):1464 - 1475, 2008. Remote Sensing Data Assimilation Special Issue.

[8] O. Titaud, A. Vidard, I. Souopgui, and F.-X. L. Dimet. Assimilation of image sequences in numerical models. Tellus A, 62:30-47, 2010. 